\title{
An Efficient Cluster Head Selection Algorithm for Wireless Sensor Networks -Edrleach
}

\author{
Ebin Deni Raj \\ (Mtech IT, VIT University, India)
}

\begin{abstract}
The Cluster-head Gateway Switch Routing protocol (CGSR) uses a hierarchical network topology. CGSR organizes nodes into clusters, with coordination among the members of each cluster entrusted to a special node named cluster-head. The cluster head selection is done with the help of any of the algorithm for cluster head selection. Energy is the primary constraint on designing any Wireless Networks practically. This leads to limited network lifetime of network. Low-Energy Adaptive Clustering Hierarchy (LEACH) and LEACH with deterministic cluster head selection are some of the cluster head algorithms that enable to optimize power consumption of WSN. There are various factors like density \& distance, threshold based, power efficient. Load balancing and scalability are the other factors which plays important role in the selection of Cluster head. Algorithms based on load balancing reduce communication cost to a great extent. The algorithms that this study is focused are A Density and Distance based Cluster Head, An Energy Efficient Algorithm for Cluster-Head Selection in WSNs, Consumed Energy as a Factor for Cluster Head. These three algorithms are analyzed and studied in this paper. The analysis of these algorithms gave birth to a new algorithm called EDRLEACH, which is proposed through this paper.
\end{abstract}

Keywords-Cluster head,energy efficient algorithms, selection head algorithms, wireless sensor,networks.

\section{Introduction}

To compare three cluster head selection algorithm to LEACH algorithm and analyze the same to propose a new algorithm

\subsection{Objective}

A wireless network which consisting of spatially distributed autonomous devices using sensors to cooperatively monitor physical or environmental conditions .It mainly consist of very large number of small, relatively inexpensive and low-power sensors which are connected to the wireless network. Wireless Sensor Networks (WSNs), have a wide range of applications in a variety of fields including physiological monitoring, environmental applications, military applications and home

Security [1]. Because of the fact that each node has only limited energy resource and the battery power and it is practically not possible to replace battery every now and then. However, the energy constraint is unlikely to be solved soon due to slow progress in developing battery capacity. So what that can be done is to find some efficient algorithm which can use energy for a larger lifetime.

The average number of sensors in a wireless sensor network is 500 to 1000.The goal is to minimize the energy spent for delivering a packet from source to destination is reduced. Energy efficient sensors can increase the lifetime of the whole sensor network system to a great extent. Various clustering techniques are present which reduce energy consumption. One of the most well-known clustering approach, which is widely used, is the LEACH (Low Energy Adaptive Clustering Hierarchy). In a wireless sensor network the cluster head can change from time to time depending on various factors such as mobility, energy density and distance between nodes. This paper is basically a study of three different algorithms namely A Density and Distance based Cluster Head, An Energy Efficient Algorithm for Cluster-Head Selection in WSNs, Consumed Energy as a Factor for Cluster Head. [2]

\section{Related Work}

The algorithms that are under study that led to the new algorithm are

1) A Density and Distance based Cluster Head Selection Algorithm in Sensor Networks

2) An Energy Efficient Algorithm for Cluster-Head Selection in WSNs

3) Consumed Energy as a Factor for Cluster Head Selection in Wireless Sensor Networks

The Density and Distance based Cluster Head Selection Algorithm divides the cluster area into two perpendicular diameters in such a way that four quadrants are got. Each node in each quadrant is able to select a cluster head depending on the number of nodes in that quadrant and the distance from its 'would be' cluster head. This is the basic DDHS scheme that is used in wireless sensor networks.[1]

Energy Efficient Algorithm for Cluster-Head Selection is basically a modification of LEACH algorithm. Although there are numerous algorithms which came up saying modification of LEACH, this is 
supposed to be more energy efficient and distribute energy load more evenly. Each node in the network decides whether or not to become a cluster-head for the current round based on the suggested percentage of clusterheads for the network and the number of times the node has been a cluster-head so far. The node will choose a random number between zero and one, if the number is less than a threshold,the node becomes a cluster-head for the current round.[2]

Consumed Energy as a Factor for Cluster Head Selection is the third algorithm that comes under this study. It has a modified threshold formula for balanced Cluster Head Selection Algorithm to increase the life time and decrease the power consumption of Wireless Sensor Networks. This is also a modified version of LEACH. The LEACH algorithm is broken into two rounds and each round begins with set-up phase and followed by a steady state phase.[3]Setup phase does organize the clusters while steady phase deals with the data transfer to the base station.

\section{Problem Definition}

All the algorithms that are being discussed is compared to LEACH algorithm.LEACH scheme considers a scenario where a large number of sensors in a field want to communicate with a base-station that is far from them. It makes use of an adaptive rotating cluster-head scheme which determines which nodes communicate with the base-station. Rotating cluster-heads are used based on the fact that fixed cluster-heads will die first because of direct communication. In each round a node chooses to become a cluster-head independent of other nodes. Once chosen it becomes ineligible for the next $1 / \mathrm{P}$ rounds where $\mathrm{P}$ is the desired percentage of cluster-heads in the network.

Initially when clusters are being created each node decides whether or not to become a cluster-head for the present round .This decision is based on the suggested percentage of clustered for the network (determined a priori) and the number of times the node has been a cluster-head so far. This decision is made by the node $\mathrm{n}$ choosing a random number between 0 and 1 . If the number is less than a threshold value $T$ (n), the node becomes a cluster-head for the current round. The threshold is set as:

$$
T(n)= \begin{cases}\frac{P}{1-P *(\operatorname{rmod} \not)} & \text { if } \mathrm{n} \in \mathrm{G} \\ 0 & \text { otherwise }\end{cases}
$$

Fig :3.1

Where $\mathrm{P}=$ the desired percentage of cluster-heads (e.g. $\mathrm{P}=0.5$ ), $\mathrm{r}=$ the current round, and $\mathrm{G}$ is the set of nodes that have not yet been cluster-heads in the last $1 / \mathrm{p}$ rounds. Using this threshold every node will be a cluster-head at some point within $1 / \mathrm{p}$ rounds.

\subsection{Limitations of LEACH}

1) Not efficient for large-scale networks.

2) Fixed percentage of cluster-heads for any size network (5\%).

3) The protocol may lead to concentration of cluster-heads in one area of the network.

4) It assumes that all nodes can communicate over one hop (directly) with the base station.

5) Uniform energy dissipation assumed for both cluster-heads and other nodes in any given round.

6) All nodes start with equal energy residual levels.

The below discussed algorithms rectify the problems in LEACH algorithm through one way or the other. Network life time is the major concern in all the algorithms here. Usually network lifetime defined as the time span from the deployment to the instant when the network is considered nonfunctional. When a network should be considered nonfunctional is, however, application-specific. In this case it is important to know when the first sensor dies. So this paper limits the definition of network lifetime as the instant when the first sensor dies.[4]

The algorithms under discussion are as follows

\subsection{A Density and Distance based Cluster Head Selection Algorithm in Sensor Networks}

The each steps of proposed DDCHS algorithm are described as follows

[stepl]Local Grouping divides cluster area into two perpendicular diameters to get four quadrants.

[step2]Compare the node density that is the number of cluster members in each quadrant and select candidate quadrants.

[step3]Compare the node distance that is from the nearest cluster head in candidate quadrants and select cluster head.

Following cluster head determination, the nodes that are picked as cluster heads advertise their status to their immediate neighbors, i.e., each cluster head communicates with neighboring devices by transmitting a signal identifying the particular node as a cluster head and inviting these neighbors to join in the cluster. Other 


\section{An Efficient Cluster Head Selection Algorithm For Wireless Sensor Networks -Edrleach}

nodes may also participate in the advertising process. Hearing these advertisement messages, each sensor node chooses the nearest cluster head and registers itself as a cluster member leading to the formation of clusters. [1] Simulation results verify that the DDCHS algorithm extend the lifetime of network 50\% more than the LEACH algorithm.

\subsection{An Energy Efficient Algorithm for Cluster-Head Selection in WSNs}

There are various network models for WSNs. For the development of our method, some reasonable assumptions about the sensor nodes are made. These assumptions are similar to those incorporated in and are as follow:

(i) All the sensor nodes are homogeneous, stationary and energy constrained.

(ii) The base station is a high-energy node, located far away from the sensor nodes.

(iii) All the nodes can transmit with enough power to reach to the base station if needed.

(iv) Nodes always have data to send to the end user and nodes located close to each other, have correlated data.[2]

This algorithm proposes an indirect solution to solve the problem of even energy distribution without getting information from other nodes. The differentiation of each node's energy can be used as a parameter to judge about that node and its neighboring nodes. In our method, each node decides independently to be a clusterhead in the next round (i.e. round $\mathrm{r}+1$ ), by monitoring variation of its energy consumption between current and previous round (i.e. in round $\mathrm{r}$ and $\mathrm{r}-1$ ). Suppose $\Delta-1$ and $\Delta$ are, respectively, energy consumption of the node in round $\mathrm{r}-1$ and $\mathrm{r}$. If $\Delta-1<\Delta$ (i.e. energy consumption of the node in the current round be larger than that of the previous round), this means that in the round $r$, the cluster-head acted against this node (and all of its neighboring nodes) as it had to consume more energy for its data transmission. So, for a more even distribution of energy load, it is logical that in the next round, cluster-head be chosen in this region of the network to alleviate its high energy consumption during previous round. To make this point practical, we add another condition to the previous condition of cluster-head selection. The added condition is simply considering the difference between $\Delta$ and $\Delta-1$, if $\Delta-1<\Delta$, then it can compete to be a cluster-head in the next round (round $\mathrm{r}+1)$. In this case, the threshold $\mathrm{T}(\mathrm{n})$ is modified and it is given by:

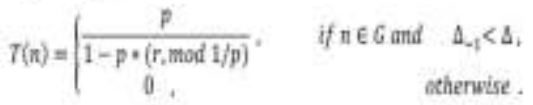

Fig.4.1Formula for threshold

The rational behind this method is its awareness about the choosing of the cluster-head position. The reason is that if cluster-head is near some nodes in a round it will not be near them in the next round. In the first two rounds, our model works like LEACH. Each node memorizes its energy level in the two previous rounds so can compute $\Delta l$ and $\Delta 2$ with subtracting them.

This algorithm distributes the energy even more efficiently. The simulation results show that this algorithm can improve system lifetime more than 10\%.[5]

\subsection{Consumed Energy as a Factor for Cluster Head Selection in Wireless Sensor Networks}

The formula mentioned in Fig: 1.1 is modified for this algorithm. The rotation of cluster heads and the metric of residual energy are not sufficient to balance the energy consumption across the network .Once a node becomes a cluster head in a certain round, its initial energy decreases because it uses energy to transmit data to BS. In order not to be cluster head in the same round, its threshold value must be less than a random number between zero and one. Otherwise it has the chance to die shortly. That leads the life time of the whole network to decrease. Hence it is necessary to incorporate its consumed energy as a component in threshold formula of cluster head selection to decrease its threshold value. The inclusion of consumed energy, a new approach, as extended factor to reduce threshold increases Life time better than residual energy. The proposed formula is:

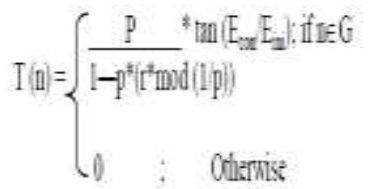

Fig4.2 Formula for threshold energy

- It enables to reduce the threshold T (n) without network stuck.

- Nodes with less consumed energy will more likely become cluster heads than nodes with more consumed energy in a given round.

- With longer distance between the base station and the nodes, network lifetime has no significant change.

- It can be achieved a remarkable increase in network life time. 
- It can be even applied for dense wireless sensor nodes. The new proposed threshold formula for LEACH has a considerable change in the network life time. Simulations reveal that this algorithm can increase the network life time to $47-575 \%$.

\begin{tabular}{|l|l|l|l|}
\hline Network Life Time & DDCH & Energy efficient & Consumed energy \\
\hline PEACH & $\begin{array}{l}\text { Remains almost the } \\
\text { same with any number } \\
\text { of nodes }\end{array}$ & $\begin{array}{l}\text { Increases when the } \\
\text { initial energy is very } \\
\text { large }\end{array}$ & $\begin{array}{l}\text { Remains same with } \\
\text { increase in number of } \\
\text { nodes and distance } \\
\text { between nodes and BS } \\
\text { (to a limit) }\end{array}$ \\
\hline Advantage & $\begin{array}{l}\text { Suited for sensor } \\
\text { system involving } \\
\text { mobile nodes }\end{array}$ & $\begin{array}{l}\text { Very practical and easy } \\
\text { to implement }\end{array}$ & $\begin{array}{l}\text { Even if the distance } \\
\text { between base station } \\
\text { and node is really high, } \\
\text { it has no significant } \\
\text { effect in network life. } \\
\text { Network will never get } \\
\text { stuck }\end{array}$ \\
\hline Disadvantage & $\begin{array}{l}\text { Node interaction result } \\
\text { in energy loss }\end{array}$ & $\begin{array}{l}\text { First two rounds } \\
\text { similar to LEACH }\end{array}$ & $\begin{array}{l}\text { Some nodes will be } \\
\text { the cluster head for } \\
\text { very long time and } \\
\text { some may not get the } \\
\text { chance to be the cluster } \\
\text { head }\end{array}$ \\
\hline Application (eg:) & Disaster recovery & $\begin{array}{l}\text { Forest fire detection } \\
\text { system }\end{array}$ & $\begin{array}{l}\text { Military applications } \\
\text { like intruder detection }\end{array}$ \\
\hline
\end{tabular}

Table 1: Comparison of Algorithms with LEACH algorithm

The algorithms DDCH, energy efficient and consumed energy efficient are compared to the most currently used algorithm - LEACH, and the best one is found to be consumed energy as a factor for cluster head selection.[6]

\section{Proposed Changes}

Keeping in mind the need to limit all sorts of communication overhead and thereby reducing the overall cost some changes have been suggested below to overcome most of the limitations as seen in the base LEACH implementation.

A general network layout is constructed at the base-station by determining the neighbors of all the nodes in the first round. Along with this list the residual energy of each node (limitation 6) as well as distance from base-station too is stored which is then used to determine cluster heads at the start of every round. Though this may seem to be rather expensive for the nodes, this procedure is carried out only once i.e. during the initialization of the network and all subsequent rounds require aggregation of the node residual energies ONLY. [7]

The node-linkage table for an m-sized sensor network is therefore a ' $\mathrm{m} \times \mathrm{m}$ ' bit-field matrix where 1 denotes presence of a link and 0 otherwise. As the matrix is symmetric this can further be halved for memory considerations. The cluster heads for the subsequent round are selected based on the weightages assigned to the parameters: no. of neighbors, residual energy and distance to BS. A sorting of the table reduces the search from an $\mathrm{O}\left(\mathrm{n}^{2}\right)$ order to $\mathrm{O}(\mathrm{n})$. The sort is performed on the basis of residual energy (ranges) and then on the number of neighbors in each range. After selection of cluster heads with elimination of common neighbor nodes to prevent closeness (limitation 3). This ensures uniform node population below each cluster head. As can be seen above, the number of cluster heads selected in each round is dynamic as opposed to the static methodology in the LEACH protocol (limitation 2). Each round ends when $50 \%$ or more of the cluster heads reach a predetermined threshold energy value. This is followed by the cluster heads informing the BS of the status of the current residual energy of all the nodes which it is currently supervising. The selection of cluster head is then carried out as before.[8]

\section{A)EDRLEACH algorithm}

Keeping in mind the need to limit all sorts of communication overhead and thereby reducing the overall cost some changes have been suggested below to overcome most of the limitations as seen in the base LEACH implementation.

A general network layout is constructed at the base-station by determining the neighbors of all the nodes in the first round. Along with this list the residual energy of each node (limitation 6) as well as distance from base- 


\section{An Efficient Cluster Head Selection Algorithm For Wireless Sensor Networks -Edrleach}

station too is stored which is then used to determine clusterheads at the start of every round. Though this may seem to be rather expensive for the nodes, this procedure is carried out only once i.e. during the initialization of the network and all subsequent rounds require aggregation of the node residual energies ONLY.

The node-linkage table for an m-sized sensor network is therefore an ' $\mathrm{m} \mathrm{x} \mathrm{m}$ ' bit-field matrix where 1 denotes presence of a link and 0 otherwise. As the matrix is symmetric this can further be halved for memory considerations. The cluster-heads for the subsequent round are selected based on the weightages assigned to the parameters: no. of neighbors, residual energy and distance to BS. A sorting of the table reduces the search from an $\mathrm{O}\left(\mathrm{n}^{2}\right)$ order to $\mathrm{O}(\mathrm{n})$. The sort is performed on the basis of residual energy (ranges) and then on the number of neighbors in each range. After selection of cluster-heads with elimination of common neighbor nodes to prevent closeness (limitation 3) a backtrack of the now hopefuls is done so that an equal distribution of nodes is assigned to each cluster-head. This ensures uniform node population below each cluster-head.

As can be seen above, the number of cluster-heads selected in each round is dynamic as opposed to the static methodology in the LEACH protocol (limitation 2). Each round ends when 50\% or more of the clusterheads reach a pre-determined threshold energy value. This is followed by the cluster-heads informing the BS of the status of the current residual energy of all the nodes which it is currently supervising. The selection of cluster-head is then carried out as before.[8]

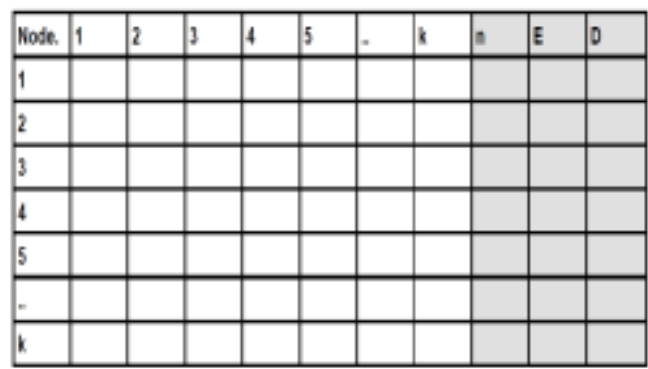

\section{B) Changes Proposed}

Fig 5.1: the ned table for k nodes

\section{1) First Round}

a )Initialization: BS sends out an initsignal along with the TX range to use for finding neighbors.

b) Flooding: Each node determines the neighbors in the specified TX range and sends this list back to the base station along with current residual energy along with distance.

c) Table creation $(B S)$ : The base station then compiles the data into a table as shown above of neighbors, num of neighbors (n), residual energy (E) and distance (D).

d) Selection of Cluster-heads:The cluster heads are selected depending on D, E, n. Max weightage is given to E and appropriate levels to D and $\mathrm{n}$ (mathematical model of which is to be constructed for finding the optimum weightages).

Bit-fields are created for the neighbor representation with a 1 indicating the presence of a neighbor and 0 otherwise.

e)Sorting:The cluster heads are selected sequentially depending on the sorted list. The list is updated for neighbors based on already selected cluster heads. The process continues until all the nodes have a cluster head. The "cluster-head" assignment to the rest of the nodes is done in a sequential fashion from the top of the stack of cluster heads selected. Each node is then checked against the selected cluster head list to find the nearest cluster head. If the nearest cluster head is in its transmission range then the node is assigned to that cluster for the current round. Round proceeds as in LEACH.

2)Subsequent Round(s)

a) Reinitialization:

BS sends out an init signal.

b)End round:

Each cluster head collects the residual energy of nodes under it and sends it back to BS.

c)Table updation(BS):

The base station then updates the data in the table.

d)Selection of Cluster-heads

The Procedureis similar to in LEACH.

3)Suppositions

No memory / computing / energy constraints for the base-station.

Each node can determine its residual energy level as a scalar quantity.Each node can directly communicate with the base-station 


\section{Conclusion}

The suggested new protocol EDRLEACH is based on clustering with maximum lifetime for wireless sensor networks. It improves LEACH by using a very equally distributed cluster and decreasing the unequal topology of the clusters. The new network protocol can be built on the shortcomings of Leach to try and rectify them.The applications of the new algorithm are immense as the life period has increased considerably.

\section{Journal Papers}

\section{References}

[1] MohammadJavadHajikhani, BahmanAbolhassani, "n Energy Efficient Algorithm for Cluster-Head Selection in WSNs, 2010 5th International Symposium on Telecommunications (IST'2010).

[2] Kyounghwa Lee, Joohyun Lee, Hyeopgeon Lee, Yongtae Shin A Density and Distance based Cluster Head Selection Algorithm in Sensor NetworksFeb. 7-10, 2010 ICACT 2010

[3] DesalegnGetachewMelese, HuagangXiong, QiangGaoConsumed Energy as a Factor For Cluster Head Selection in Wireless Sensor Networks" 978-1-4244-3709-2/10/ 2010 IEEE

[4] Hung-Chin Jang and Hon-Chung LeeEfficient Energy Management to Prolong Lifetime of Wireless Sensor Network"L. Ying and Y. Haibin, "Energy Adaptive Cluster-Head Selection forWireless Sensor Networks, Proceedings of the Sixth IEEEInternational Conference on Parallel and Distributed Computing,Applications and Technologies, Dec.2005

[5] Sungju Lee, Jangsoo Lee, Jangsoo Lee,Hongjoong, Sin, SeunghwanYoo, Sanghyuck Lee, Jaesik Lee, Yongjun Lee, and Sungchun Kim, An Energy- Efficient Distributed Unequal Clustering Protocol for Wireless Sensor networks. World Academy of Science, Engineering \& Technology 48, 2008.

[6] John A. Stankovice, Research Challenges for Wireless Sensor Networks. July 2004.

Books

[7] Wiley Series on Wireless Communications and Mobile Computing FUNDAMENTALS OFWIRELESS SENSOR NETWORKSpublished in 2010

[8] CHAPMAN \& HALL/CRC COMPUTER and INFORMATION SCIENCE SERIESHandbook of Algorithms for Wireless Networking and Mobile Computing 2006 